This article was downloaded by:[EPFL Lausanne]

On: 20 February 2007

[EPFL Lausanne]

Access Details: [subscription number 768419380]

Publisher: Taylor \& Francis

Informa Ltd Registered in England and Wales Registered Number: 1072954

Registered office: Mortimer House, 37-41 Mortimer Street, London W1T 3J H, UK

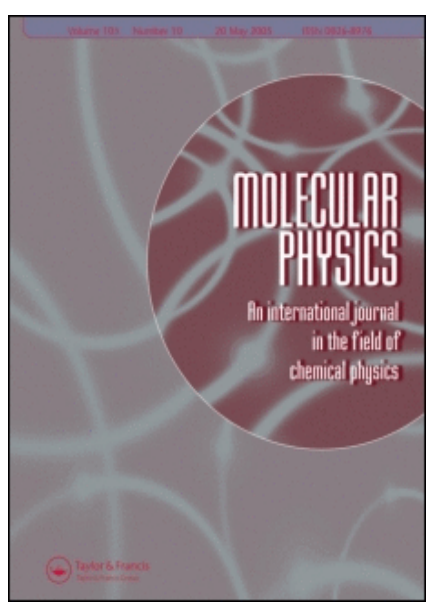

Molecular Physics An International J ournal in the Field of Chemical Physics

Publication details, including instructions for authors and subscription information: http://www.informaworld.com/smpp/title content=t713395160

Molecular dynamics study of nitrobenzene and 2-nitrophenyloctyl ether saturated with water

To link to this article: DOI: $10.1080 / 00268970601076509$

URL: http://dx.doi.org/10.1080/00268970601076509

Full terms and conditions of use: $h$ ttp://www.informaworld.com/terms-and-conditions-of-access.pdf

This article maybe used for research, teaching and private study purposes. Any substantial or systematic reproduction, re-distribution, re-selling, loan or sub-licensing, systematic supply or distribution in any form to anyone is expressly forbidden.

The publisher does not give any warranty express or implied or make any representation that the contents will be complete or accurate or up to date. The accuracy of any instructions, formulae and drug doses should be independently verified with primary sources. The publisher shall not be liable for any loss, actions, claims, proceedings, demand or costs or damages whatsoever or howsoever caused arising directly or indirectly in connection with or arising out of the use of this material.

(c) Taylor and Francis 2007 


\title{
Molecular dynamics study of nitrobenzene and 2-nitrophenyloctyl ether saturated with water
}

\author{
M. JORGE, R. GULABOSKI $\uparrow$, C. M. PEREIRA and M. N. D. S. CORDEIRO* \\ Universidade do Porto, Portugal
}

(Received 19 July 2006; revised 18 October 2006)

\begin{abstract}
In this paper, results obtained from molecular dynamics (MD) simulations on the watersaturated organic liquids nitrobenzene (NB) and 2-nitrophenyloctyl ether (NPOE) are presented. Both solvents are extremely important in many areas of chemistry, and they are seen as the most promising successors of n-octanol for ion transfer studies at the liquid-liquid interface. Thermodynamic (mass density, enthalpy of vaporization), structural (radial distribution functions) and dynamic (diffusion coefficients) properties of both liquids have been calculated and compared with those obtained from MD simulations of the corresponding pure (water-free) organic liquids. The relatively polar nature of NB allows for an efficient incorporation of water into the organic liquid, minimizing the impact on the solvent structure and dynamics. NPOE exhibits added hydrophobicity due to the presence of a long aliphatic chain, and this has an effect on its water solvation properties. Steric effects caused by these chains, together with the much slower dynamic response of the solvent, substantially hinder the motion of water and prevent interactions with other water molecules. The different behaviour of these solvents towards water provides a good means for studying the properties of these liquids at the interface with water.
\end{abstract}

\section{Introduction}

Currently, there is a dire need for an adequate choice of organic liquid solvent to be employed in processes that take place across the interface separating two immiscible liquids. The nature of the organic solvent seems to govern the processes of solute and charge (ion or electron) transfer across liquid-liquid interfaces [1-4], thus influencing a variety of interfacial phenomena, such as extraction [1], adsorption [1], metal-ion complexation $[1,3,5]$, interfacial reactions $[1-3,6]$, interactions between bio-active ions and phospholipids [4, 7], drug permeation through biomembranes [4] and phasetransfer catalysis [1]. As well as respecting all health, safety and environmental requirements, the organic solvents used in these processes should also possess well-balanced ion solvation properties, fine-tuned to allow for the successful accomplishment of a particular task. Ion solvation properties are usually improved when the solvent molecules include a polar group, which

*Corresponding author. Email: ncordeir@fc.up.pt

†On leave from the Department of Chemistry, Faculty of Natural Sciences and Mathematics, 1000 Skopje, Macedonia normally translates into a higher dielectric constant of the liquid medium. Perhaps the worthiest of such organic solvents are those that exhibit a degree of amphiphilic behaviour, i.e. organic solvents that promote the coexistence of very different environments, polar and non-polar, in direct proximity $[1,3,4]$, the most ubiquitous of which is n-octanol [8]. n-octanol has been used for the determination of partition coefficients of a huge number of neutral organic solutes, and is seen as an ideal proxy for phospholipids (which are the major constituents of biological membranes). [1, 4, 8] However, it is inadequate for ion transfer studies across liquid-liquid interfaces, since its interface with water cannot be polarized by applying a potential difference, as is usually done in classical electrochemical set-ups [6].

The most promising successors of n-octanol for ion transfer studies at the water-organic interface are nitrobenzene (NB) [1-3, 6] and, recently, 2-nitrophenyln-octyl ether (NPOE) [3, 5, 9-15]. Both solvents have low miscibility with water and high dielectric constants, which make them good media for solvation of ionic species. The greatest advantage of NPOE is that it is not known to show any toxic effects on humans. Due to the 

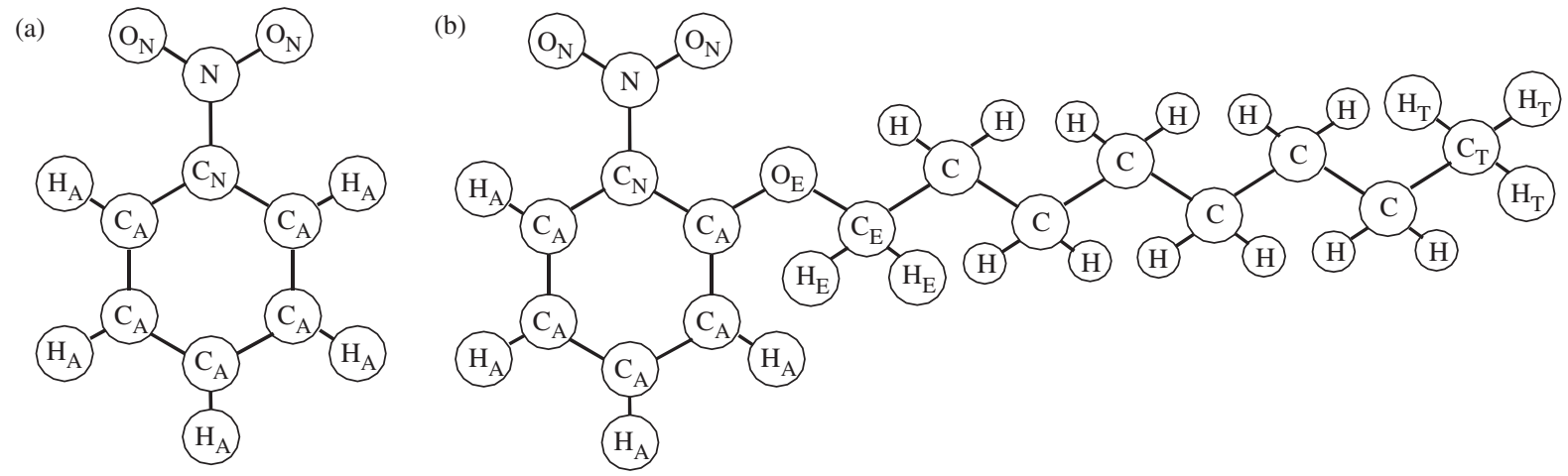

Figure 1. Schematic diagram of the (a) NB and (b) NPOE molecular models, showing the labels used for the atom types.

importance of these solvents in chemistry, there is no question that computer simulations of these systems will be of great help for a proper understanding of their solvation features. We have recently presented a comprehensive molecular dynamics study of pure NB and NPOE, including the calculation of thermodynamic, structural and dynamic properties of the bulk liquids, as well as the solvation of potassium and chloride ions [16]. However, a crucial aspect that is missing is a detailed study of the interactions of these solvents with water. Although in small amounts, both solvents contain water dissolved in them - in laboratory experiments, they are usually saturated with water. It is worth stressing that the water present in some organic solvents can also play a significant role in the solvation properties of those solvents, as has been recently proven by studies of n-octanol $[3,9,10,17]$. In this work, we present results of molecular dynamics simulations of both NB and NPOE organic solvents saturated with water. Emphasis is put on the effect of dissolved water on thermodynamic and dynamic properties of the solvents, as well as on the structure of the water solvation shell. This supplements our previous work [16] in providing a standpoint for comparison with properties of the water-organic interfaces, which are currently being simulated at our laboratory.

\section{Simulation methods and potentials}

NB and NPOE were simulated using the all-atom OPLS force-field [18] with fixed bond lengths, flexible angles and dihedrals, as well as improper dihedrals to keep the aromatic rings planar. A nitrobenzene potential model developed by Michael and Benjamin (MB) [19] was also tested. Previously, we compared this model to OPLS and found it to yield slightly worse agreement with experiments for all calculated properties, except for the molecular dipole moment [16]. Since this property may have a strong influence on the interactions between NB and water (a very polar molecule), we have chosen to compare the performance of both models in simulations of the saturated liquid. Figure 1 shows schematic diagrams of the NB and NPOE molecules, giving the labels used for the atoms. Water was represented by the SPC/E model [20], with fixed bond lengths and angle.

Molecular dynamics (MD) simulations of the saturated liquids were performed using version 3.3 of the GROMACS software package [21,22]. The equations of motion were integrated with the Verlet leap-frog algorithm [23]. By constraining all bond lengths using the LINCS algorithm [24], we were able to employ time steps of $2 \mathrm{fs}$. The simulations were carried out in cubic boxes, with periodic boundary conditions in the three Cartesian directions. Long-range electrostatic interactions were computed using the particle-mesh Ewald method [25]. The real-space part of the Ewald sum and the short-range Lennard-Jones $(\mathrm{L}-\mathrm{J})$ interactions were calculated up to a cut-off distance of $1.1 \mathrm{~nm}$ with the help of a neighbour list, updated every 10 time steps. In addition, $\mathrm{L}-\mathrm{J}$ interactions between 1.1 and $1.5 \mathrm{~nm}$ were calculated every 10 steps and added to the total energy, and a long-range dispersion correction was applied to both energy and pressure. The temperature was held fixed at $298 \mathrm{~K}$ using the Nosé-Hoover thermostat $[26,27]$ and the pressure was kept constant at 1 bar by the Parrinello-Rahman coupling scheme [28]. Both schemes employed a coupling constant of $1.0 \mathrm{ps}$.

Our objective in this paper is to study the saturated organic liquids under conditions at which they are employed experimentally. Therefore, we have opted to reproduce in our simulations the 'experimental' solubility of water in each solvent. It should be noted, however, that this does not necessarily correspond to the solubility of 'model' water in 'model' organic solvent. A precise determination of these solubilities is beyond the scope of this paper. As such, one must calculate the number of water molecules that need to be included in the 
Table 1. Thermodynamic properties of pure and saturated NB and NPOE liquids.

\begin{tabular}{lccc}
\hline Solvent & & $\rho\left(\mathrm{kg} \mathrm{m}^{-3}\right)$ & $\Delta H_{\mathrm{vap}}\left(\mathrm{kJ} \mathrm{mol}^{-1}\right)$ \\
\hline Nitrobenzene (OPLS) & pure & $1174.0 \pm 0.8^{\mathrm{a}}$ & $54.25 \pm 0.40^{\mathrm{a}}$ \\
& saturated & $1173.1 \pm 1.2$ & $55.30 \pm 0.41$ \\
Nitrobenzene (MB) & pure & $1163.9 \pm 1.3^{\mathrm{a}}$ & $48.84 \pm 1.40^{\mathrm{a}}$ \\
NPOE (OPLS) & saturated & $1163.6 \pm 1.1$ & $46.72 \pm 1.40$ \\
& pure & $1037.2 \pm 0.9^{\mathrm{a}}$ & $105.32 \pm 0.85^{\mathrm{a}}$ \\
& saturated & $1034.6 \pm 1.0$ & $105.18 \pm 0.65$ \\
\hline
\end{tabular}

${ }^{\mathrm{a}}$ Ref. [16].

simulation boxes to match the experimental equilibrium solubility of water in both solvents. The solubility of water in $\mathrm{NB}$ at $298 \mathrm{~K}$ is $0.2 \mathrm{~mol} / \mathrm{L}$ [12], which means one water molecule for every 49 nitrobenzene molecules, while the water solubility in NPOE is only $0.046 \mathrm{~mol} / \mathrm{L}$ [12], yielding $1 \mathrm{H}_{2} \mathrm{O}$ for every 90 NPOE. Taking these numbers, we have prepared three cubic boxes, one containing two water molecules plus 180 NPOE molecules and two containing five water molecules plus $245 \mathrm{NB}$ molecules (one for each potential model). The water molecules were inserted randomly in previously equilibrated pure-solvent boxes. The whole systems were re-equilibrated for $500 \mathrm{ps}$, followed by production runs of $2 \mathrm{~ns}$, which were divided into $200 \mathrm{ps}$ blocks for averaging purposes. The dissolved water diffusion coefficients calculated from the above simulations suffer from large statistical errors. To improve the statistics, we have performed an additional $5 \mathrm{~ns}$ run for each model solvent, containing 10 dissolved water molecules. Of course, these runs no longer reflect the experimental water solubility in the organic liquids. The impact of the added water concentration on the results will be analysed below.

The properties of the saturated liquids are best analysed by taking the pure liquids as reference points. For that purpose, we have performed simulations of pure SPC/E water in the NPT ensemble, using the same parameters as above, except for smaller cut-off radii $(1.0 \mathrm{~nm}$ for the short range and $1.2 \mathrm{~nm}$ for the long range). Cubic boxes containing different numbers of water molecules $(N=113,267,533,901$ and 1433) were simulated so as to account for the system-size dependence of self-diffusion coefficients [29]. MD simulations of pure NB and NPOE have been performed previously and are presented elsewhere [16].

\section{Results and discussion}

\subsection{Thermodynamics and structure}

The calculated values for the density $(\rho)$ and enthalpy of vaporisation $\left(\Delta H_{\mathrm{vap}}\right)$ of the saturated liquids are compared to the values obtained from simulations of the pure liquids [16] in table 1 . The values of $\Delta H_{\text {vap }}$ were calculated following the Jorgensen approach [30], assuming that the sum of the vibrational terms with the kinetic energy is equal in the gas and liquid phases. The densities of the saturated liquids are all lower than the corresponding pure components, but this difference is small for NPOE and almost negligible for NB (in fact, the densities of pure and saturated NB are equal within statistical error). Nevertheless, it is interesting to note that the variation in density is largest for NPOE, even though this solvent contains less water than NB. In other words, a smaller amount of water causes more disruption to the structure of NPOE. The reasons for this effect will become apparent below, when we analyse the structure of the water solvation shell. In accordance with the previous observation, the solvent-solvent radial distribution functions (RDF) of saturated NB are indistinguishable from the pure component RDFs, while in the case of NPOE there are small but noticeable differences (results not shown). The enthalpies of vaporization show also small differences, but there is no clear trend in the variation.

Before examining the interactions between dissolved water and the organic solvents, we will look at the interactions between water molecules themselves. Starting with nitrobenzene, figure 2 shows the oxygenoxygen $\left(\mathrm{O}_{\mathrm{W}}-\mathrm{O}_{\mathrm{W}}\right)$ and oxygen-hydrogen $\left(\mathrm{O}_{\mathrm{W}}-\mathrm{H}_{\mathrm{W}}\right)$ RDFs for water in saturated NB. The $\mathrm{O}_{\mathrm{W}}-\mathrm{O}_{\mathrm{W}}$ RDFs show a strong peak at approximately the same distance $(0.275 \mathrm{~nm})$ as in pure SPC/E water, but the second peak is hardly discernible. Integration of the first peak yields an estimate of the average number of neighbours in the first coordination shell. This number is 0.6 for the OPLS model and 1.05 for the MB model, which are much lower than the coordination number of 4.45 obtained in the pure water simulations. Naturally, the upper limit of the coordination number in NB is 4 , since there are only 5 water molecules in the simulation box. The low 


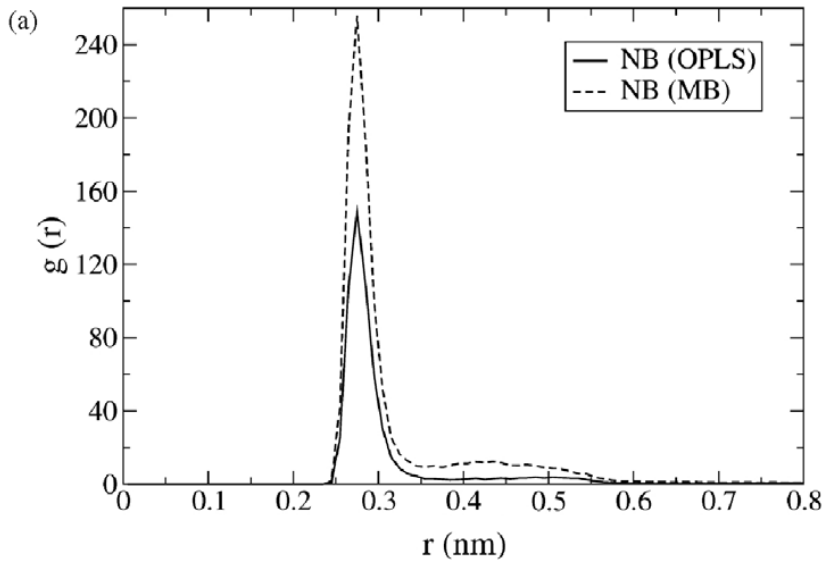

(b)

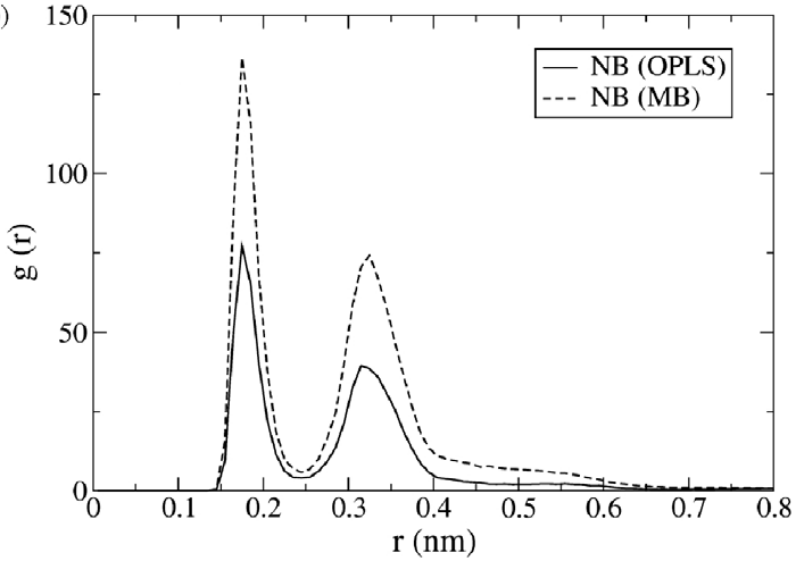

Figure 2. $\mathrm{O}_{\mathrm{W}}-\mathrm{O}_{\mathrm{W}}$ (a) and $\mathrm{O}_{\mathrm{W}}-\mathrm{H}_{\mathrm{W}}$ (b) radial distribution functions for water in NB and NPOE liquids.

coordination numbers observed are primarily a consequence of the reduced density of water in the saturated nitrobenzene solutions.

The shape of the $\mathrm{O}_{\mathrm{W}}-\mathrm{H}_{\mathrm{W}}$ RDFs is also qualitatively similar to that of pure SPC/E water (first peak at about $0.175 \mathrm{~nm}$, second peak at about $0.32 \mathrm{~nm}$ ). However, the second peak in NB is approximately half the height of the first peak, while in pure water these heights are almost identical. This reflects a strong depletion of the second coordination shell of water. Integration of the first peak of the $\mathrm{O}_{\mathrm{W}}-\mathrm{H}_{\mathrm{W}}$ RDF yields an average of 0.14 oxygens per hydrogen for the OPLS potential and 0.25 for the MB potential, compared to 0.94 for pure water. Considering that a water hydrogen atom can only form one hydrogen bond, these numbers mean that almost all hydrogens in pure water are hydrogen-bonded to water oxygens $(94 \%)$, but in NB this percentage drops to between 14 and $25 \%$. Another interesting quantity is the probability that a water molecule in the first coordination shell participates in the solvation as a hydrogenbond donor. Such probabilities can be obtained from

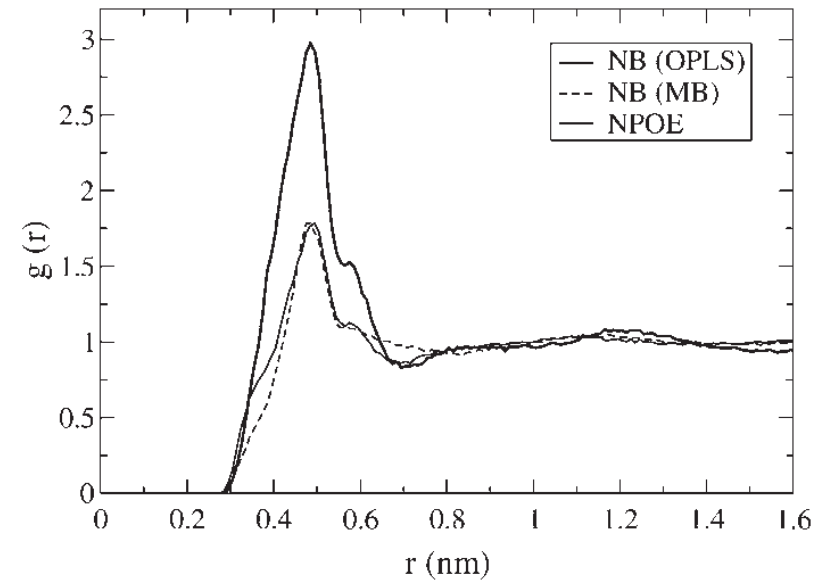

Figure 3. $\mathrm{O}_{\mathrm{W}}-\mathrm{C}_{\mathrm{N}}$ radial distribution functions for saturated NB and NPOE liquids.

the previous numbers, and are $48 \%$ for OPLS, $47 \%$ for $\mathrm{MB}$ and $42 \%$ for pure water. It is also worth noting that the water-water rdfs obtained from the simulations with 10 dissolved water molecules (see section 2) are qualitatively similar, with a slightly increased coordination number, due to the higher water concentration (results not shown).

The picture emerging from these numbers is that of a robust water structure, bearing a strong resemblance to that of pure water, but incomplete due to the significant reduction in density - the second solvation shell is practically absent while the first shell is restricted to small transient clusters of water molecules formed inside the organic liquid. These observations are consistent with previous work on saturated 2-heptanone [31]. The MB model allows for more interactions between water molecules, most likely due to its lower density and viscosity. As for NPOE, the water RDFs show no significant interactions between water molecules up to very large distances. In fact, visual inspection of simulation snapshots shows that water molecules dissolved in NPOE remain isolated from each other during the length of the run. This is most likely due to the restricted mobility of dissolved water molecules, brought about by the very high viscosity and slow dynamic response of NPOE [9-16]. As we will see below, this will also have a strong effect on the water diffusion coefficient.

Moving on to the water-organic interactions, figure 3 shows the $\mathrm{O}_{\mathrm{W}}-\mathrm{C}_{\mathrm{N}}$ RDFs. The $\mathrm{C}_{\mathrm{N}}$ atom is a good reference point since it lies very close to the centre of mass of the NB molecule. The RDFs for the three solvents show a single, very broad peak at approximately $0.49 \mathrm{~nm}$. Integration of this peak yields 7.5 OPLS nitrobenzene molecules surrounding each water 


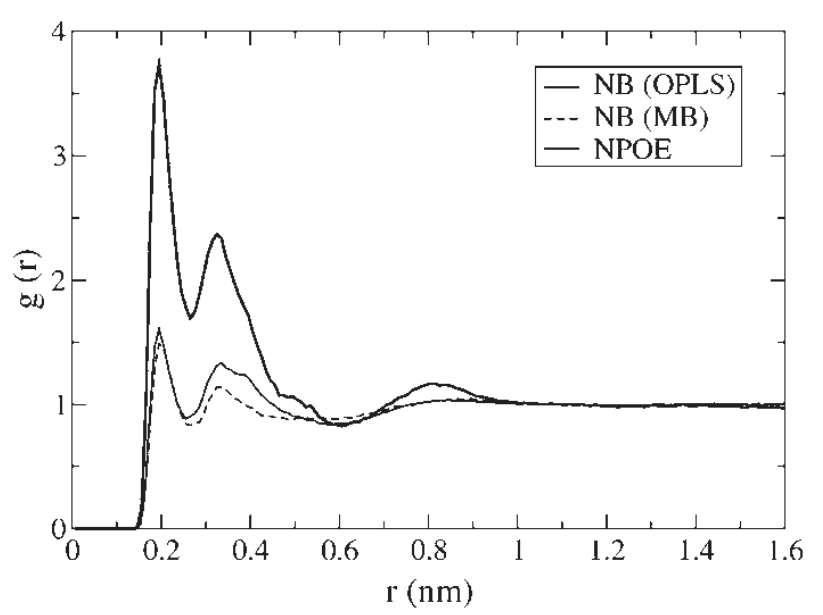

Figure 4. $\mathrm{H}_{\mathrm{W}}-\mathrm{O}_{\mathrm{N}}$ radial distribution functions for saturated NB and NPOE liquids.

molecule, 7.3 MB nitrobenzene molecules and only 4.6 NPOE molecules. The latter number is much lower due to steric hindrances caused by the aliphatic chains, which prevent a higher number of nitrobenzene rings (the polar part of the NPOE molecule) from packing around the water molecule.

These RDFs give us an idea about the global solvation shell of water molecules in the organic solvents. To have a more precise idea of the local interactions between water and organic molecules, we plot the $\mathrm{H}_{\mathrm{W}}-\mathrm{O}_{\mathrm{N}}$ RDFs in figure 4. This RDF shows a strong first peak at $0.195 \mathrm{~nm}$ and a secondary peak at about $0.325 \mathrm{~nm}$. The shape is similar to the $\mathrm{O}_{\mathrm{W}}-\mathrm{H}_{\mathrm{W}}$ RDF (figure 2b) but slightly shifted to larger distances, and reflects hydrogen bonding between the water hydrogens and the oxygens of the nitro group. These bonds can also be inferred from additional RDFs involving water and the other atoms of the nitro group (data not shown). Integration of the first peak of the RDFs in figure 4 yields a total of $0.77 \mathrm{O}_{\mathrm{N}}$ atoms per water hydrogen for OPLS nitrobenzene, 0.73 for $\mathrm{MB}$ nitrobenzene and 0.74 for NPOE.

The $\mathrm{O}_{\mathrm{W}}-\mathrm{H}_{\mathrm{A}}$ RDFs are plotted in figure 5, and show a strong peak at approximately $0.28 \mathrm{~nm}$. These interactions are of a similar nature to those known to occur between $\mathrm{O}_{\mathrm{N}}$ and $\mathrm{H}_{\mathrm{A}}$ atoms in pure NB and NPOE [16]. Integrating the first peak of the RDFs in figure 5 gives seven aromatic hydrogens per water hydrogen in the case of NB and four in the case of NPOE. Once more, the smaller number of neighbours in NPOE is caused by steric effects owing to the presence of the aliphatic chains.

Taking these results together with those for the waterwater interactions (figure 2), one can get a more complete picture of the solvation shell of water

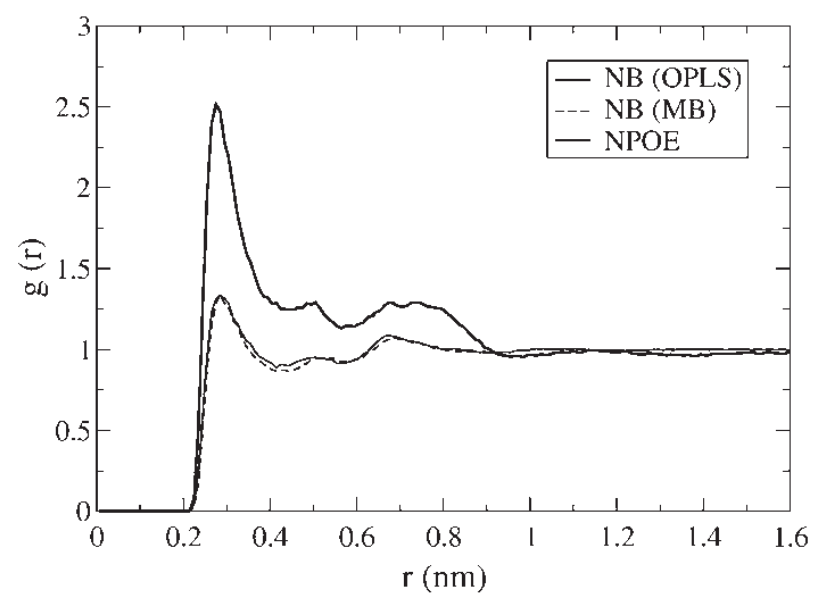

Figure 5. $\mathrm{O}_{\mathrm{W}}-\mathrm{H}_{\mathrm{A}}$ radial distribution functions for saturated NB and NPOE liquids.

molecules in both organic solvents. When water is dissolved in NB the majority of $\mathrm{H}_{\mathrm{W}}$ atoms (73 to $77 \%$ ) are hydrogen-bonded to nitro groups of solvent molecules, a few (between 14 and 25\%) are bonded to other water molecules and only a very small fraction ( 2 to $9 \%$ ) are not taking part in any hydrogen bond. The latter fraction is comparable to that of pure water (about 6\%), which means that water dissolved in NB behaves as a hydrogen donor in much the same way as pure water. On the contrary, it behaves differently as a hydrogen acceptor - even though there are interactions between $\mathrm{O}_{\mathrm{W}}$ atoms and aromatic hydrogens in the solvent, these are weaker and occur at much larger distances than the hydrogen bonds in pure water. Nevertheless, these interactions are similar to those between the opposite ends of the NB dipole [16], and thus the water molecule can be easily incorporated into the solvent structure without causing much disruption, which explains the almost negligible change in thermodynamic properties when going from pure to saturated NB (table 1). The water solvation shell in NB is illustrated in figure 6(b), which shows a snapshot obtained during the MD simulation by selecting only the solvent molecules that are within the water solvation shell. Both the $\mathrm{H}_{\mathrm{W}}-\mathrm{O}_{\mathrm{N}}$ and the $\mathrm{O}_{\mathrm{W}}-\mathrm{H}_{\mathrm{A}}$ interactions can be seen in this figure (e.g. NB molecules on the bottom and on the left, respectively). In this particular case, two water molecules are hydrogen-bonded to each other forming a transient dimer.

The structure of NPOE is much more disrupted by the presence of dissolved water than that of NB. Even though there is a similar proportion of $\mathrm{H}_{\mathrm{W}}-\mathrm{O}_{\mathrm{N}}$ hydrogen bonds, the remainder $(26 \%)$ of water hydrogens in saturated NPOE are not participating in any hydrogen bonds. Similarly, there are less $\mathrm{O}_{\mathrm{W}}-\mathrm{H}_{\mathrm{A}}$ 

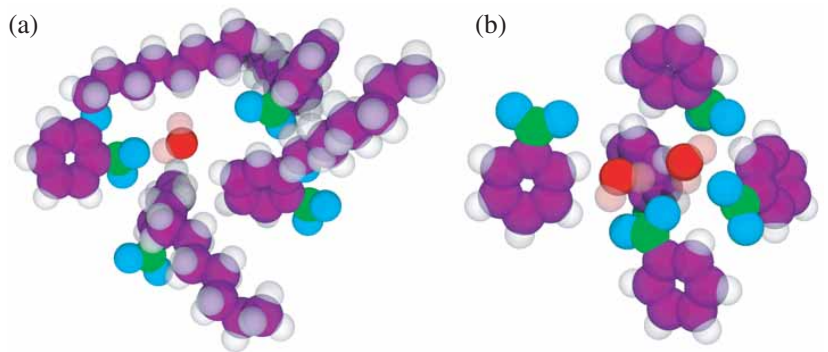

Figure 6. Snapshots of the water solvation shell in NPOE (a) and NB (b). The water oxygens are represented by solid red spheres, while the water hydrogens are the transparent red spheres. The remaining spheres are solvent atoms: $\mathrm{C}-$ purple; $\mathrm{N}$ - green; $\mathrm{O}$ - blue; $\mathrm{H}$ - white (transparent). Some solvent molecules have been removed for better visualization.

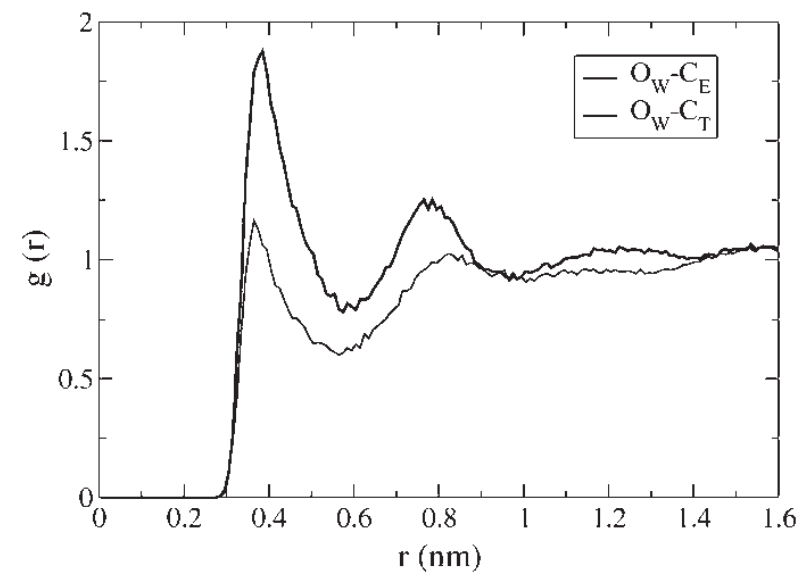

Figure 7. Radial distribution functions between $\mathrm{O}_{\mathrm{W}}$ atoms and carbons on both ends of the NPOE chain.

interactions in NPOE than in NB, and these are again weaker than the $\mathrm{O}_{\mathrm{W}}-\mathrm{H}_{\mathrm{W}}$ bonds. This disruption is manifested in the larger density decrease of saturated NPOE relative to the pure liquid (table 1) and is illustrated in figure 6(a). The $\mathrm{H}_{\mathrm{W}}-\mathrm{O}_{\mathrm{N}}$ and $\mathrm{O}_{\mathrm{W}}-\mathrm{H}_{\mathrm{A}}$ interactions are once again evident (e.g. NPOE molecules on the left and bottom, respectively), but the aliphatic chains prevent a more efficient packing of the solvent around the water molecule - the packing is much looser than in figure 6(b). The steric hindrances caused by the aliphatic chains are also evident in figure 7 , which shows the RDFs between the water oxygens and the terminal $\left(\mathrm{C}_{\mathrm{T}}\right)$ and ether $\left(\mathrm{C}_{\mathrm{E}}\right)$ carbons of the NPOE chain. Even though the $\mathrm{C}_{\mathrm{T}}$ atom is more accessible, water preferentially interacts with the $\mathrm{C}_{\mathrm{E}}$ atom, since this is closer to the polar part of the molecule.

\subsection{Diffusion coefficients}

The diffusion coefficients of water and organic solvents in the saturated liquids $\left(D_{\text {Sat }}\right)$ were obtained from $5 \mathrm{~ns}$ runs containing 10 water molecules each (see section 2), by linear fits to the mean square displacement of each component, following the Einstein relation. These are compared to the self-diffusion coefficients of the respective pure liquids ( $D_{\text {Pure }}$ ) in table 2 . We have tested for possible artifacts in the diffusion results arising from the use of a thermostat and barostat, by performing NVT and NVE runs for a few test cases. The results obtained in the three ensembles are statistically indistinguishable, and we can confidently say that the dynamics of our NPT simulations are free from any artifacts. This is to be expected, since pressure and temperature coupling algorithms based on the extended system approach (such as the Nose-Hoover and Parinello-Rahman methods) have been shown to yield a correct representation of the system dynamics [32].

The self-diffusion coefficient of pure water is plotted in figure 8 as a function of inverse box length. In agreement with previous studies [16, 29], a linear relationship is observed, which can be extrapolated to infinite box size to estimate the macroscopic selfdiffusion coefficient.

The diffusion coefficients of the saturated liquids must also be corrected for finite-size effects. Since performing the same type of calculation as for the pure components would be too computationally demanding, we use here a simplified approach: the diffusion coefficient obtained in a finite cubic box is extrapolated to infinite box size by applying the same slope as in the pure solvent. This procedure assumes that the diffusion of all species in a saturated solution is dominated by the dynamics of the solvent. We believe that this is a reasonable assumption given that the fraction of water is minute in all cases under study here. All values shown in table 2 have been corrected for finite-size effects.

Even though $D_{\text {Sat }}$ for the organic solvents is always higher than $D_{\text {Pure, }}$ the values are within statistical error of each other. This means that the presence of a small amount of water does little to change the solvent dynamics. On the contrary, the diffusion of water in the saturated liquids is reduced relative to that of pure water; this reduction is more pronounced, the higher the viscosity of the solvent. Nitrobenzene is only slightly more viscous than water (between 1.4 and 1.7 times [16]) and thus the water molecules can retain a high diffusion coefficient (albeit lower than in pure water). During a given MD run, the water molecules explore most of the box volume, interacting with NB molecules and/ or with other water molecules (see figure $6 \mathrm{~b}$ ). In NPOE, however, the much higher solvent viscosity (about 17 times that of pure water [16]) causes the motion of water molecules to be strongly coupled to 
Table 2. Diffusion coefficients of water, NB and NPOE in pure and saturated liquids.

\begin{tabular}{lccc}
\hline Component & & $D_{\text {Sat }}\left(10^{-9} \mathrm{~m}^{2} \mathrm{~s}^{-1}\right)$ & $D_{\text {Pure }}\left(10^{-9} \mathrm{~m}^{2} \mathrm{~s}^{-1}\right)$ \\
\hline Nitrobenzene (OPLS) & organic & $0.973 \pm 0.037$ & $0.945 \pm 0.045^{\mathrm{a}}$ \\
& water & $1.035 \pm 0.127$ & $2.785 \pm 0.019$ \\
Nitrobenzene (MB) & organic & $1.048 \pm 0.086$ & $1.030 \pm 0.087^{\mathrm{a}}$ \\
& water & $1.529 \pm 0.213$ & $2.785 \pm 0.019$ \\
NPOE (OPLS) & organic & $0.072 \pm 0.013$ & $0.068 \pm 0.020^{\mathrm{a}}$ \\
& water & $0.292 \pm 0.068$ & $2.785 \pm 0.019$ \\
\hline
\end{tabular}

${ }^{\mathrm{a}} \mathrm{OW}-\mathrm{OW}$, and ${ }^{\mathrm{b}} \mathrm{OW}-\mathrm{HW}$ radial distribution functions for water in NB.

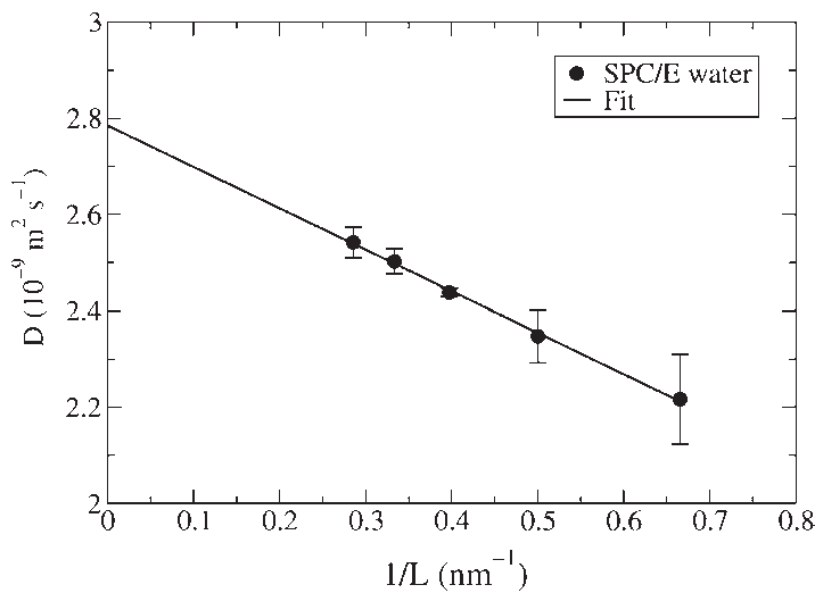

Figure 8. Self-diffusion coefficient of pure SPC/E water as a function of inverse box length. The line is a linear fit to the data, extrapolated to infinite box size.

the motion of the organic molecules, and thus the water diffusion coefficient is significantly lower than in pure water. Water molecules migrate very little during an MD run, and are always surrounded by NPOE molecules (see figure 6a). Comparing the results for the two different nitrobenzene models, we see that they also follow a decreasing trend with solvent viscosity - MB is less viscous than OPLS [16] and thus water shows a higher diffusion coefficient in the first solvent.

Finally, it is worth commenting on the effect of increased concentration of water in the organic liquids. The dissolved water diffusion coefficients calculated from runs at the experimental solubility are $1.261 \pm 0.307$ for OPLS nitrobenzene, $1.601 \pm 0.451$ for MB nitrobenzene and $0.376 \pm 0.088$ for NPOE. The values shown in table 2 are all slightly lower than these, but the difference is within statistical error. Thus, we are unable at this point to clarify whether this is a meaningful trend or simply a consequence of the poor statistics of the calculations with low water concentration.

\section{Conclusions}

In this paper, we have presented a detailed study of saturated nitrobenzene and NPOE liquids, which are important solvents in interfacial transfer processes. The presence of dissolved water in the amounts observed experimentally induces only small changes in the thermodynamic and dynamic properties of the solvents. These changes are more prominent for NPOE than for $\mathrm{NB}$, due to differences in their water solvation properties. The relatively polar nature of NB provides this solvent with the ability to interact efficiently with water molecules, via hydrogen bonds between water hydrogens and nitro-group oxygens (as well as, less significantly, by weaker interactions between water oxygens and aromatic hydrogens). The $\mathrm{H}_{\mathrm{W}}-\mathrm{O}_{\mathrm{N}}$ bonds account for the majority of interactions involving water molecules, the remainder taking place with other water molecules. These polar interactions allow for water to be easily incorporated into the solvent, minimizing the disruption to the liquid structure and properties. NPOE, on the other hand, possesses a polar part identical to NB and a rather large non-polar part. Even though $\mathrm{H}_{\mathrm{W}}-\mathrm{O}_{\mathrm{N}}$ hydrogen bonds are still observed in this liquid, steric effects caused by the aliphatic chains prevent an efficient packing of NPOE molecules in the water solvation shell. The overall effect of this is an increased disruption of the solvent structure and properties, relative to the pure liquid.

As for the water structure, it shows high robustness, retaining many characteristics of bulk water behaviour, but depleted due to the large reduction in density. In $\mathrm{NB}$, water molecules are allowed to move rather freely, and thus their diffusion coefficient in the solvent is not much lower than in bulk. Contrastingly, the high viscosity of NPOE and the hydrophobic nature of the aliphatic chains hinder the motion of water molecules, essentially 'trapping' them inside the solvation shell. As a consequence, water motion is significantly coupled to that of the solvent, and thus exhibits much lower diffusion coefficients than in bulk. 
The solvents examined here form an interesting pair for studies of liquid-liquid interfaces and ion transfer across these interfaces. We have shown in previous simulation work that NPOE and NB are thermodynamically similar but are quite different from the point of view of dynamic response [16]. In this work, we have shown that the presence of the hydrophobic aliphatic chain in NPOE changes the liquid's behaviour towards water, relative to that of NB. By comparing these two solvents, one might be able to determine the effects of this increased hydrophobicity on the properties of aqueous interfaces and on the ion transfer process. Work towards this end is currently underway in our laboratory.

\section{Acknowledgements}

This work was supported by Fundação para a Ciência e a Tecnologia (FCT), Projects POCTI/QUI/42402/2001 and POCTI/QUI/41074/2001. R.G. thanks the FCT for the post-doctoral fellowship SFRH/BPD/14894/2004.

\section{References}

[1] A. G. Volkov, Liquid Interfaces in Chemical, Biological and Pharmaceutical Applications (Marcel Dekker, New York, 2001), Vol. 95. Chapter 2.

[2] Y. Marcus, Ion Properties (Marcel-Dekker, New York, 1997).

[3] F. Scholz, U. Schroeder, and R. Gulaboski, Electrochemistry of Immobilized Particles and Droplets (Springer, Heidelberg/Berlin, 2005).

[4] B. Testa, H. van de Waterbeemd, G. Folkers, and R. Gay, Pharmacokinetic Optimization in Drug Research (WileyVCH, Weinheim, Germany, 2001). Chapter 6.

[5] C. M. Pereira, N. Tirilly, M. C. Martins, and F. Silva, Fresenius J. Anal. Chem. 369, 609 (2001).

[6] H. H. Girault and D. J. Schiffrin, in Electroanalytical Chemistry, edited by A. J. Bard (Marcel-Dekker, New York, 1989), Vol. 15.

[7] R. Gulaboski, C. M. Pereira, M. N. D. S. Cordeiro, I. Bogeski, E. Ferreira, D. Ribeiro, M. Chirea, and A. F. Silva, J. Phys. Chem. B 109, 12549 (2005).

[8] A. Leo, C. Hansch, and D. Elkins, Chem. Rev. 71, 525 (1971).
[9] R. Gulaboski, A. Galland, G. Bouchard, K. Caban, A. Kretschmer, P. A. Carrupt, H. H. Girault, and F. Scholz, J. Phys. Chem. B 108, 4565 (2004).

[10] F. Scholz and R. Gulaboski, Chem. Phys. Chem. 6, 16 (2005).

[11] O. Valent, J. Koryta, and M. Panoch, J. Electroanal. Chem. 226, 21 (1987).

[12] Z. Samec, J. Langmaier, and A. Trojanek, J. Electroanal. Chem. 409, 1 (1996).

[13] S. Wilke and T. Zerihun, J. Electroanal. Chem. 515, 52 (2001).

[14] Z. Samec, J. Langmaier, A. Trojanek, E. Samcova, and J. Malek, Anal. Sci. 14, 35 (1998).

[15] H. J. Lee and H. H. Girault, Anal. Chem. 70, 4280 (1998).

[16] M. Jorge, R. Gulaboski, C. M. Pereira, and M. N. D. S. Cordeiro, J. Chem. Phys. B 110, 12530, accepted for publication (2006).

[17] (a) G. Bouchard, A. Galland, P. A. Carrupt, R. Gulaboski, V. Mirceski, F. Scholz, and H. H. Girault, Phys. Chem. Chem. Phys. 5, 3748 (2003); (b) R. Gulaboski, V. Mirceski, and F. Scholz, Electrochem. Commun. 4, 277 (2002).

[18] W. L. Jorgensen and J. Tirado-Rives, J. Am. Chem. Soc. 110, 1657 (1988).

[19] D. Michael and I. Benjamin, J. Electroanal. Chem. 450, 335 (1998).

[20] H. J. C. Berendsen, J. R. Grigera, and T. P. Straatsma, J. Phys. Chem. 91, 6269 (1987).

[21] H. J. C. Berendsen, D. van der Spoel, and R. van Drunen, Comput. Phys. Commun. 91, 43 (1995).

[22] E. Lindahl, B. Hess, and D. van der Spoel, J. Mol. Mod. 7, 306 (2001).

[23] R. W. Hockney and S. P. J. Goel, J. Comput. Phys. 14, 148 (1974).

[24] B. Hess, H. Bekker, H. J. C. Berendsen, and J. G. E. M. Fraaije, J. Comput. Chem. 18, 1463 (1997).

[25] U. Essman, L. Perela, M. L. Berkowitz, T. Darden, H. Lee, and L. G. Pedersen, J. Chem. Phys. 103, 8577 (1995).

[26] S. Nosé, Mol. Phys. 52, 255 (1984).

[27] W. G. Hoover, Phys. Rev. A 31, 1695 (1985).

[28] M. Parrinello and A. Rahman, J. Appl. Phys. 52, 7182 (1981).

[29] I. C. Yeh and G. Hummer, J. Phys. Chem. B 108, 15873 (2004).

[30] W. L. Jorgensen and J. Tirado-Rives, J. Am. Chem. Soc. 106, 6638 (1984).

[31] P. A. Fernandes, M. N. D. S. Cordeiro, and J. A. N. F. Gomes, J. Phys. Chem. 103, 1176 (1999).

[32] P. H. Hunenberger, Adv. Polym. Sci. 173, 105 (2005). 